\title{
INTERAÇÕES MEDICAMENTOSAS ASSOCIADAS A FITOTERÁPICOS FORNECIDOS PELO SISTEMA ÚNICO DE SAÚDE
}

\section{Drug Interactions Associated with Herbal Provided by the Unified Health System}

Rafaela Dutra Felten ${ }^{1}$, Karen Magnus ${ }^{2}$, Lidiane dos Santos ${ }^{3}$, Alessandra Hubner de Souza 4

1 Farmacêutica graduada pela Universidade Luterana do Brasil (ULBRA).

${ }^{2}$ Nutricionista. Especialista em Farmacologia e Toxicologia. Mestre e Doutoranda em Biologia Celular e Molecular pela Pontifícia Universidade Católica do Rio Grande do Sul (PUCRS).

${ }^{3}$ Farmacêutica. Docente e coordenadora do curso de Farmácia da Universidade Luterana do Brasil (ULBRA). Especialista em Homeopatia. Mestre em Ciências Farmacêuticas.

${ }^{4}$ Farmacêutica. Docente do curso de Farmácia da Universidade Luterana do Brasil (ULBRA). Mestre em Ciências Biológicas (Bioquímica toxicológica) e Doutora em Farmacologia Bioquímica e Molecular.

\section{Endereço para correspondência:}

Alessandra Hubner de Souza

Universidade Luterana do Brasil, Av. Farroupilha, oㅡ 8001, Bairro São José

CEP: 92425-900, Canoas, Rio Grande do Sul, Brasil.

Fone geral: 55 (51) 3477-4000 Fax: 55 (51) 3477-1313

E-mail: alessandrahubnersouza@gmail.com 


\title{
Resumo
}

Devido a variadas comprovações científicas relatando os benefícios de plantas medicinais, tem se observado um aumento na utilização da fitoterapia no tratamento de várias doenças. O Brasil ganha destaque nesse campo possuindo um terço da flora mundial. Os fitoterápicos possuem compostos químicos responsáveis pelos seus variados efeitos. Entretanto, muitas dessas substâncias ativas ainda não têm suas atividades farmacológicas bem descritas ou conhecidas. Os conhecimentos científicos gerados a partir de ensaios clínicos randomizados e a descrição de casos clínicos têm apontado a relação de efeitos adversos, toxicidade e contraindicações relacionadas ao uso concomitante de fitoterápicos e medicamentos alopáticos, ainda que o medicamento fitoterápico seja considerado de baixa toxicidade. Diretrizes brasileiras do Ministério da Saúde determinaram prioridades na investigação de plantas medicinais e implantaram a fitoterapia na prática de atenção básica. Portanto, o objetivo deste estudo foi realizar um levantamento na literatura científica sobre as interações decorrentes do uso de medicamentos associados aos fitoterápicos do Sistema Único de Saúde e, com isso, auxiliar na divulgação dos dados existentes, fortalecendo o conhecimento de profissionais da área da saúde sobre os efeitos medicinais e tóxicos das plantas e, consequentemente colaborar com a implementação das práticas de fitoterapia.

Palavras-chave: Medicamentos Fitoterápicos; Sistema Único de Saúde; Efeitos Colaterais e Reações Adversas Relacionadas a Medicamentos; Plantas Medicinais.

\begin{abstract}
Due to numerous scientific evidences reporting the benefits of medicinal plants it has been observed an increase in the use of phytotherapy in the treatment of various diseases. Brazil stands out in this field having a third of the world's flora. Phythoterapics are constituted of chemical compounds responsible for their varied effects. However, many of these active substances do not have their pharmacological activities described or well known yet. The scientific knowledge generated from randomized clinical trials and description of clinical cases has pointed out the relationship of adverse effects,
\end{abstract}




\section{Revisão}

\section{Atenção à Saúde}

toxicity and contraindications related to the concomitant use of phytotherapics and allopathic drugs, even though phytoteraphics are considered to have low toxicity. Guidelines of the Brazilian Ministry of Health determined priorities in research of medicinal plants and implemented phytotherapy in the practice of primary care. Therefore, the aim of this study was to survey the literature on the interactions caused by the associated use of phytoterapics and drugs from Unified Health System and thereby assist in the dissemination of existing data strengthening the knowledge of health professionals about medicinal and toxic effects of plants, and consequently contribute to the implementation of phytotherapy practices.

Keywords: Phytotherapeutic Drugs; Unified Health System; Drug-Related Side Effects and Adverse Reactions; Medicinal Plants.

\section{INTRODUÇÃO}

De acordo com a legislação, fitoterápico é todo o "medicamento obtido empregando-se exclusivamente matérias-primas ativas vegetais. É caracterizado pelo conhecimento da eficácia e dos riscos de seu uso, assim como pela reprodutibilidade e constância de sua qualidade. Sua eficácia e segurança é validada através de levantamentos etnofarmacológicos de utilização, documentações tecnocientíficas em publicações ou ensaios clínicos de fase 3. Não se considera medicamento fitoterápico aquele que, na sua composição, inclua substâncias ativas isoladas, de qualquer origem, nem as associações destas com extratos vegetais"1.

As plantas medicinais podem ser de grande valia como terapia alternativa para auxiliar no tratamento de diversas doenças. A implantação de programas para o uso de fitoterápicos na atenção primária à saúde está relacionada a alta demanda de medicamentos, em todas as regiões do país ${ }^{2,3}$.

O Brasil ganha destaque por possuir um terço da flora mundial (tendo a Amazônia a maior diversidade de espécies vegetais com possível ação medicinal) e, com isto, o desenvolvimento de produtos fitoterápicos ganha destaque no cenário científico farmacológico 3,4 
Os medicamentos fitoterápicos são compostos por diversos componentes químicos presentes nas plantas, os quais podem ser responsáveis pelos efeitos farmacológicos. Esses efeitos são explicados pela interdependência única destas substâncias, podendo ser antagônicos e/ou sinérgicos, ocorrendo como resultado da interação dos diversos constituintes químicos ativos. Entretanto, grande parte das moléculas ativas (responsáveis pelas atividades farmacológicas dos medicamentos fitoterápicos) presentes nestes compostos são desconhecidas. Isto pode aumentar a possibilidade de interações quando fármacos são utilizados juntamente com fitoterápicos ${ }^{5,6}$.

Os conhecimentos gerados com a condução de ensaios clínicos randomizados e a descrição de diversos casos clínicos na literatura científica mostram que as plantas medicinais e os produtos fitoterápicos podem provocar vários efeitos adversos, toxicidade e apresentar contraindicações, principalmente, quando associados a medicamentos alopáticos ${ }^{7,8}$. Isso aponta para a necessidade de cautela na utilização tanto de ervas medicinais quanto de fitoterápicos.

Interações entre fármacos e fitoterápicos podem causar alterações nas concentrações plasmáticas dos fármacos, bem como mudanças nos perfis de eficácia e segurança. Essas interações podem interferir na farmacocinética e farmacodinâmica dos medicamentos. Quando se faz o uso concomitante de fitoterápicos e fármacos, pode-se ter um efeito farmacológico inesperado, que acarreta em redução ou aumento desse efeito ${ }^{5,7,9}$. O uso inadequado de um fitoterápico - considerado equivocadamente pela população como um medicamento de baixa toxicidade - pode desencadear reações adversas graves quando em uso concomitante com outros medicamentos ${ }^{10-}$ 12.

Apesar disto, com base em achados científicos e conhecimento popular (em relação à utilização de plantas medicinais) e pela grande demanda por terapias alternativas que possam auxiliar na manutenção e/ou recuperação dos indivíduos usuários do Sistema Único de Saúde (SUS) ${ }^{13}$, o Governo Federal do Brasil implantou a Política Nacional de Plantas Medicinais e Fitoterápicos da qual se originou o Programa Nacional de Plantas Medicinais e Fitoterápicos (PNPMF) ${ }^{14}$. Diretrizes brasileiras do Ministério da Saúde (MS) determinaram prioridades na investigação de plantas medicinais implantando a fitoterapia como prática da medicina, orientando 


\section{Revisão}

\section{Atenção à Saúde}

comissões de saúde a buscarem a inclusão no SUS². O MS através da Portaria $\mathrm{n}^{\circ}$ 971 de 3 de maio de 2006, disponibiliza opções terapêuticas aos usuários do SUS, entre essas o uso de plantas medicinais e fitoterápicos ${ }^{15}$.

A falta de informação sobre as propriedades dos fitoterápicos e os possíveis riscos decorrentes do seu uso juntamente com medicamentos alopáticos, pode elevar as chances da ocorrência de reações adversas, com consequente dano à saúde do indivíduo. Com base no exposto, o objetivo deste trabalho, foi realizar uma busca de informações farmacológicas dos fitoterápicos fornecidos pelo SUS e suas principais interações com medicamentos. Esta revisão visa fornecer embasamento científico para profissionais da saúde auxiliando a implementação da prática segura de fitoterapia no SUS.

\section{METODOLOGIA}

O presente estudo teve por finalidade investigar e compilar as possíveis interações entre medicamentos versus fitoterápicos fornecidos pelo SUS. Para isto, realizou-se um estudo exploratório do tipo revisão bibliográfica a partir das bases de dados Scielo, Medline e Pubmed. As referências foram datadas a partir do ano 2000 (por serem ainda escassas as referências) e os termos usados para a seleção da bibliografia do estudo foram: medicamentos fitoterápicos, Sistema Único de Saúde, efeitos colaterais e reações adversas relacionadas a medicamentos, plantas medicinais, além do nome das plantas (alcachofra, aroeira, babosa, cáscara-sagrada, espinheira-santa, garra-do-diabo, guaco, hortelã, isoflavona-de-soja, plantago, salgueiro e unha-de-gato).

\section{RESULTADOS}

\section{ALCACHOFRA (Cynara scolymus L.)}

Uso Terapêutico: Extratos de alcachofra são utilizados com o intuito de tratar pacientes com hipercolesteremia e dispepsia ${ }^{16,17}$. 
Interações Medicamentosas: Existem poucos ensaios clínicos realizados para investigar o efeito hipocolesterolêmico dessa planta, sendo assim não foi encontrada documentação sobre possíveis interações medicamentosas com medicamentos dislipidêmicos ${ }^{8}$. Já, em estudos pré-clínicos com roedores, foi verificado que o efeito diurético da alcachofra pode apresentar sinergia quando ela é usada concomitante aos diuréticos. Como resulado desta interação pode ocorrer queda de pressão arterial por hipovolemia e aumento da excreção de potássio com risco de hipocalemia. Essas interações associadas aos diuréticos foram identificadas como as mais graves estando entre os fármacos citados os diuréticos de alça (ex: furosemida) e os tiazídicos (ex: hidroclorotiazida) ${ }^{14,18,19}$.

\section{AROEIRA (Schinus terebenthifolius)}

Uso Terapêutico: tem ação anti-inflamatória, antimicrobiana e cicatrizante 20,21 .

Interações Medicamentosas: Não foram encontradas referências bibliográficas sobre interações entre medicamentos e aroeira, característica que poderia estar relacionada ao fato da aroeira ter seu uso mais difundido em formulações de uso externo (tópico).

\section{BABOSA (Aloe vera)}

Uso Terapêutico: A babosa é muito usada como cicatrizante e também para queimaduras de primeiro e segundo graus (uso tópico) ${ }^{22,23}$, porém a relatos de uso oral da babosa, mas não há estudos científicos que comprovem sua real eficácia.

Interações Medicamentosas: apresenta interação moderada com hipoglicemiantes, diuréticos, sevoflurano, laxantes e varfarina (quando em uso oral).

Tem interação grave com digoxina (fármaco usado na insuficiência cardíaca), podendo seus efeitos serem potencializados por hipocalemia. A babosa usada com a digoxina causa hipermineralocorticismo que reduz os níveis séricos de potássio, fator predisponente da intoxicação digitálica ${ }^{24}$. Tem ainda interação com ervas contendo 
glicosídeos cardíacos, ervas ou suplementos com potencial hipoglicemiante, cavalinha, alcaçuz e ervas laxantes ${ }^{19}$.

Em relação aos corticosteróides, a associação foi relacionada à hipertensão e arritmia cardíaca, bem como redução do efeito imunossupressor do fármaco ${ }^{5}$.

Segundo Abebe ${ }^{25}$ o consumo da babosa juntamente com medicamentos pode reduzir a absorção do fármaco. Além disto, pode ocorrer efeito sinérgico com fármacos hipoglicemiantes (antidiabéticos), bupropiona, clorpromazina, fluoxetina e propanolol ${ }^{5}$, com aumento no risco de hipoglicemia. Com diuréticos tiazídicos e de alça e corticóides pode ocorrer efeito aditivo por causa da perda de potássio, levando à hipocalemia. Com laxativos, a babosa potencializa o efeito laxativo ${ }^{5,26}$.

\section{CÁSCARA-SAGRADA (Rhamnus purshiana)}

Uso Terapêutico: Usada para constipação ocasional ${ }^{14}$.

Interações Medicamentosas: O seu uso juntamente com diuréticos tiazídicos e de alça não é recomendado, podendo ocorrer perda de potássio em excesso, resultando em quadro de hipocalemia ${ }^{19,24,26,27}$. Outro aspecto importante é a promoção do desequilíbrio de eletrólitos, podendo potencializar o efeito de glicosídeos cardiotônicos. Como a cáscara-sagrada intensifica o trânsito gastrintestinal, aumentando o peristaltismo, pode ainda afetar a absorção de água, eletrólitos e medicamentos administrados por via oral e aumentar a pressão sanguínea ${ }^{26,27}$.

Também há relatos de hiperatividade cardiovascular e toxicidade cardiovascular por redução dos níveis de potássio, quando a cáscara-sagrada é usada junto com corticosteróides ${ }^{5,25,26}$ e, quando usada em associação com a digoxina pode causar consequente redução dos níveis séricos de potássio ${ }^{28}$.

\section{ESPINHEIRA-SANTA (Maytenus ilicifolia)}

Uso Terapêutico: Usada para auxiliar no tratamento de gastrite e úlceras ${ }^{29}$.

Interações Medicamentosas: De acordo com um estudo experimental realizado por Montanari e Bevilacqua $^{30}$ a espinheira-santa poderia ter uma propriedade estrogênica causando perda embrionária. Esse dado indica cautela no 
uso concomitante com anticoncepcionais. De acordo com Rossato e colaboradores ${ }^{31}$ não foi encontrado interações medicamentosas com a espinheira-santa.

\section{GARRA-DO-DIABO (Harpagophytum procumbens)}

Uso Terapêutico: Usada como anti-inflamatório em osteoartrite, gota e outras condições inflamatórias ${ }^{32}$.

Interações Medicamentosas: A garra-do-diabo possui interações com antiarrítmicos, anticoagulantes, ácido acetilsalicílico, anti-inflamatórios não esteroidais (AINES), agentes antiplaquetários, glicosídeos cardíacos e varfarina ${ }^{32}$. Quando este fitoterápico é usado com antiácidos ou antagonistas do receptor histaminérgico H2, ele pode reduzir a eficácia desses medicamentos. Também há risco de redução da pressão arterial, devendo ser usado com cautela quando associados a antihipertensivos. Há evidências de que a garra-do-diabo quando usada com substratos do citocromo P450 (2C19) causa inibição da isoenzima, aumentando assim os níveis dos fármacos substratos, tais como inibidores da bomba de prótons, diazepam, carisoprodol, nelfinavir, AINES e losartana. Ainda não forma identificadas essas interações em humanos ${ }^{26}$.

O fitoterápico reduz a absorção de ferro (antianêmico) quando usados em associação ${ }^{33}$. Pode ocorrer potencialização da hipoglicemia quando associado à hipoglicemiante ora ${ }^{24}$. O fitoterápico potencializa o efeito anticoagulante, com risco de sangramento quando é usado concomitantemente com varfarina ${ }^{9,24,34}$.

\section{GUACO (Mikania glomerata)}

Uso Terapêutico: Expectorante e broncodilatador ${ }^{14,35}$.

Interações Medicamentosas: O extrato seco dessa planta pode interagir, sinergicamente in vitro, com alguns antibióticos como tetraciclinas, gentamicina, vancomicina e penicilina ${ }^{19,35}$. Tem interação moderada com anticoagulantes e há risco de sangramento nas coagulopatias e dengue ${ }^{19}$. 


\section{HORTELÃ (Mentha piperita)}

Uso Terapêutico: A hortelã é usada para dores de estômago, feridas (cicatrização), tratamento digestivo e cólicas (antiespasmódico), mas há relatos de seu uso para depressão, tosse, resfriado, dor no corpo, dor de cabeça, febre ${ }^{36,37}$.

Interações Medicamentosas: Estudos experimentais apontam que a absorção de ferro pelas proteínas sanguíneas foi inibida quando chás de hortelãpimenta foram administrados. É necessário tomar precaução na administração deste fitoterápico em pacientes anêmicos ou crianças ${ }^{14}$. Outro estudo mostrou que a hortelã administrada por via oral pode aumentar os níveis sanguíneos de fármacos como o felodipino e a sinvastatina ${ }^{35}$. Em outro estudo avaliando o uso do óleo de hortelã, os pesquisadores apontaram que este aumentou os níveis de ciclosporina no sangue. Porém, os efeitos em humanos não são claros. Nesse mesmo estudo, a administração tópica do óleo de hortelã com 5-fluoruracil foi capaz de intensificar a velocidade de absorção do antitumoral ${ }^{38}$.

Há relatos de que o óleo de hortelã altera a função do citocromo P450 e, consequentemente, pode alterar os níveis da concentração plasmática de fármacos administrados juntamente com o óleo. Esta interação aumenta o risco de intensificação dos efeitos dos fármacos ou potencialização das reações adversas graves. Além disso, pode apresentar interação leve com antiácidos (antagonista do receptor $\mathrm{H} 2 \mathrm{e}$ inibidores da bomba de prótons), interação moderada com ciclosporina, substratos do citocromo P450 (1A2, 2C19, 2C9, 3A4) e alteraração dos níveis dos hormônios FSH (Hormônio Folículo Estimulante), LH (Hormônio Luteinizante) e testosterona ${ }^{19}$.

Um estudo com pessoas portadoras de HIV/AIDS que faziam uso de Mentha spp. (hortelã) com os medicamentos antirretrovirais, apontou que esta ingesta simultânea poderia apresentar interações. A hortelã apresenta propriedades que poderia inibir as enzimas do citocromo P450, interferindo na biodisponibilidade e toxicidade dos medicamentos antirretrovirais ${ }^{39}$. 
Uso Terapêutico: auxilia no alívio dos sintomas do climatério ${ }^{40-42}$.

Interações Medicamentosas: A digoxina e a quinidina são substratos da glicoproteína-P, e esses flavonóides apresentam uma ação inibitória sobre essa proteína. A glicoproteína- $P$ atua limitando a biodisponibilidade oral, facilitando a excreção biliar e a eliminação renal dos fármacos citados. Assim, conclui-se que a interação entre isoflavonóides (isoflavona-de-soja) com digoxina e quinidina pode causar intoxicação através do aumento da concentração sérica dos fármacos ${ }^{43}$. A isoflavona-de-soja pode apresentar interação com estriol e/ou levotiroxina levando a um aumento do efeito do estrógeno e diminuição da absorção da tiroxina ${ }^{5,42}$.

\section{PLANTAGO (Plantago ovata Forssk)}

Uso Terapêutico: Reduz o nível de colesterol total e LDL no sangue e também pode ser usado para constipação intestinal ${ }^{44}$.

Interações Medicamentosas: Há evidências de que o plantago altera os níveis de varfarina e glicose no sangue e, por isso, deve ser usado com cautela e ser monitorado quando utilizado junto com anticoagulantes e hipoglicemiantes ${ }^{44}$. Pode afetar a absorção de cálcio e de outros fármacos, tais como antidepressivos, antiinflamatórios, diuréticos, salicilatos, tetraciclinas, nitrofurantoina, lítio, e digoxina ${ }^{44}$. A concentração do lítio é diminuída quando ele é usado em associação ao plantago ${ }^{45}$.

Um estudo, relacionou o plantago utilizado em associação com a levotiroxina sódica, em idosos, com a diminuição da absorção do fármaco ${ }^{42}$.

\section{SALGUEIRO (Salix alba)}

Uso Terapêutico: Possui ação anti-inflamatória, analgésica e antitérmica ${ }^{46-48}$. Interações Medicamentosas: Quando o salgueiro é usado juntamente com ácido acetilsalicílico e paracetamol pode ocasionar nefrotoxicidade. Deve ser acrescentado também, que o uso do paracetamol com salicilatos pode produzir efeito aditivo sobre a inibição da função plaquetária ${ }^{48}$. Quando o fitoterápico é associado 


\section{Revisão}

Atenção à Saúde

com medicamentos a base de ácido acetilsalicílico (aspirina) e anticoagulantes (varfarina) pode determinar aumento no risco de sangramento ${ }^{5,26}$. Os taninos presentes no salgueiro podem interferir na absorção do sulfato ferroso ou do ferro presente em alimentos ${ }^{48}$.

\section{UNHA-DE-GATO (Uncaria tomentosa)}

Uso Terapêutico: A unha de gato pode ser usada para o tratamento de artrite, reumatismo, abscesso, inflamação, febre, alergia, neoplasia, úlcera gástrica, contracepção, irregularidade menstrual, recuperação de parto, impurezas da pele, diabetes, disenteria, dor de cabeça, infecção bacteriana e fúngica. Possui também ação analgésica e sedativa, mas seu principal uso terapêutico é para os tratamentos de artrite e osteoartrite ${ }^{49-55}$.

Interações Medicamentosas: O uso do fitoterápico de forma concomitante com amitriptilina, buspirona, clomipramina, haloperidol, nefazodona pode causar aumento dos níveis plasmáticos, dos fármacos citados, por inibição do CYP 3A45,56. Um efeito sinérgico da anticoagulação de AINEs pode ser percebido com a ingestão de unha-de-gato - durante o tratamento com esses fármacos - aumentando o risco de sangramento ${ }^{5}$.

\section{DISCUSSÃO E CONCLUSÃO}

Este estudo apresentou as principais indicações relacionadas com os fitoterápicos fornecidos pelo SUS, bem como as possíveis interações que podem ocorrer quando são utilizados juntamente com fármacos. O uso de plantas medicinais e fitoterápicos representa uma prática bastante comum no Brasil. Mesmo assim, os conhecimentos acerca dos componentes químicos exatos e, principalmente, o efeito e toxicidade de cada fitoterápico ainda são pouco descritos. Isto se torna uma questão chave na utilização de fitoterápicos, como alternativa de tratamento no SUS, uma vez que, sem conhecimentos adequados não há como capacitar profissionais de saúde para a orientação e prescrição correta e, ainda menos, prever de forma segura e adequada, o resultado do tratamento com tais produtos. 
Em nossa busca, observou-se que dentre os 12 fitoterápicos pesquisados 11 apresentaram interações (com exceção da aroeira por ter seu uso de forma tópica) com riscos de interações que poderiam ser categorizados como sendo de leve a graves. Isso coloca os produtos fitoterápicos como alvo de vigilância farmacológica, uma vez que interações importantes, com diversas classes de fármacos, podem aumentar o risco de reações adversas e danos à saúde dos usuários.

Dentre as interações medicamentosas graves foram observadas a da alcachofra (com propriedades diuréticas) que em uso concomitantemente com diuréticos de alça ou tiazídicos resulta em hipocalemia, servindo este efeito também para a cáscara-sagrada. A espinheira-santa pode ter propriedade estrogênica com perda embrionária, devendo-se ter cautela quando usada junto com anticoncepcionais. A garra-do-diabo potencializa o efeito anticoagulante, causando risco de sangramento quando utilizada com varfarina. A babosa pode ter efeito adicional com fármacos hipoglicemiantes, com aumento no risco de hipoglicemia e, se usada com diuréticos causa hipocalemia. O salgueiro quando usado com ácido acetilsalicílico e paracetamol aumenta o risco de sangramento e nefrotoxicidade, respectivamente.

Os fitoterápicos são consumidos indiscriminadamente pela população. Grande parte desse consumo indiscriminado deve-se a falta de informações suficientes sobre esses medicamentos e pela crença, que por serem "naturais", não causam riscos, nem reações adversas e, principalmente, interações medicamentosas. São usados normalmente por automedicação ou em menor escala por prescrição médica, os quais estão aceitando e utilizando essa nova terapia. Atualmente, o Programa de Fitoterapia incentivado pelo Governo Federal indica que essa opção terapêutica é eficaz e de baixo custo, podendo (quando praticada de forma racional) trazer benefícios à população.

Um dos pontos que facilita o uso indiscriminado e sem orientação de plantas medicinais e fitoterápicos é a falta de regulamentação em relação a venda destes produtos. Ambos podem ser adquiridos facilmente e sem qualquer necessidade da apresentação de um receituário prescrito por um profissional da saúde, habilitado para tal. É comum a comercialização em mercados populares, lojas de suplementos nutricionais, farmácias, supermercados e, até mesmo, sendo vendidos através de 


\section{Revisão}

\section{Atenção à Saúde}

vendedores porta-a-porta e meios de comunicação como televisão, rádio e encartes por indivíduos sem qualquer conhecimento científico e habilitação para indicar de forma correta o uso.

Atualmente a prática de prescrição de fitoterápicos pelos profissionais da saúde, tem sido regulamentada, de forma louvável, pelos Conselhos Federais das distintas classes profissionais da saúde. Com isto, passou-se a exigir que os profissionais habilitados, a tal prática, tenham capacitação adequada, através de cursos de especialização para a prescrição de fitoterápicos. Isso assegura que a prática de medicinas alternativas no SUS possam ser seguras tanto para o prescritor quanto para o usuário.

$\mathrm{Na}$ contramão da qualificação dos profissionais da saúde está a falta de incentivo a pesquisas na área. Ainda são poucos os grupos de pesquisa que tem seu foco em estudos de investigação dos efeitos, reações adversas e interações medicamentosas com fitoterápicos. Os custos altos de pesquisa, grande variedade de plantas e fármacos são alguns dos pontos que dificultam a geração de novas informações. Ainda há grande necessidade de incentivo a pesquisas nesta área, no Brasil, para o desenvolvimento de novos conhecimentos. A capacitação de profissionais da saúde não pode ser alicerçada em conhecimentos antigos ou empíricos sobre o uso de plantas medicinais e fitoterápicos, podendo incorrer em risco à saúde da população. Portanto, a atualização do conhecimento de profissionais e estudantes da saúde são fundamentais para tornar mais segura e eficaz a implementação da prática fitoterápica no SUS.

Este trabalho se propôs a oferecer subsídios iniciais para a capacitação de profissionais da saúde acerca das possíveis interações entre fármacos e fitoterápicos fornecidos pelo SUS. Além disso, também apontou possíveis riscos que podem ser ocasionados pelas interações entre fitoterápicos e medicamentos alopáticos, mostrando que é crucial a pesquisa dos fitoterápicos para melhor avaliar as suas propriedades farmacológicas e, com isso, prevenir possíveis reações adversas relacionadas com seu uso. 


\section{REFERÊNCIAS}

1. Brasil. Consolidado de normas da COFID. In: Sanitária ANdV, editor. Brasilia: ANVISA; 2013. p. 1000.

2. Silva MIG, Gondim APS, Nunes IFS, Sousa FCF. Utilização de fitoterápicos nas unidades básicas de atenção à saúde da família no município de Maracanaú (CE). Rev bras farmacogn. 2006;16:455-62.

3. Santos HV, Pereira JM, Freitas RF, Royo VA. Caracterização laboratorial das dislipidemias e o uso de fitoterápicos. Rev multitexto. 2015;3(1):21-8.

4. França ISX, Souza JA, Baptista RS, Britto VRS. Medicina popular: benefícios e malefícios das plantas medicinais. Rev bras enferm. 2008;61:201-8.

5. Salvi RM, Magnus K. Interação fármaco-nutriente: desafio atual da farmacovigilância. Porto Alegre: Edipucrs; 2014. 152 p.

6. Mills E, Wu P, Johnston BC, Gallicano K, Clarke M, Guyatt G. Natural health product-drug interactions: a systematic review of clinical trials. Ther drug monit. 2005;27(5):549-57.

7. Alexandre RF, Bagatini $F$, Simões $\mathrm{CMO}$. Interações entre fármacos e medicamentos fitoterápicos à base de ginkgo ou ginseng. Rev bras farmacogn. 2008;18:117-26.

8. Alexandre RFA, Garcia FN, Simões CMO. Fitoterapia baseada em evidências. Parte 2. Medicamentos fitoterápicos elaborados com alcachofra, castanha-da-índia, ginseng e maracujá. Acta farm bonaer. 2005;24:310-4.

9. Izzo AA, Di Carlo G, Borrelli F, Ernst E. Cardiovascular pharmacotherapy and herbal medicines: the risk of drug interaction. Int j cardiol. 2005;98(1):1-14.

10. Cordeiro CHG, Chung MC, Sacramento LVS. Interações medicamentosas de fitoterápicos e fármacos: Hypericum perforatum e Piper methysticum. Revista Brasileira de Farmacognosia. 2005;15:272-8.

11. Amorim MFD, Diniz MFFM, Araújo MST, Pita JCLR, Dantas JG, Ramalho JA, et al. The controvertible role of kava (Piper methysticum G. Foster) an anxiolytic herb, on toxic hepatitis. Brazilian journal of pharmacognosy. 2007;17:448-54.

12. Silveira PF, Bandeira MAM, Arrais PSD. Farmacovigilância e reações adversas às plantas medicinais e fitoterápicos: uma realidade. Rev bras farmacogn. 2008;18(4):618-26. 
13. Saraiva SRGL, Saraiva HCC, Oliveira-Júnior RG, Silva JC, Damasceno CMD, Silva Almeida JRG, et al. A Implantação do Programa de Plantas Medicinais e Fitoterápicos no Sistema Público de Saúde: uma revisão de literatura. Rev inter pesq inov. 2015;1(1):1-11.

14. Brasil. Programa Nacional de Plantas Medicinais e Fitoterápicos. In: Secretaria de Ciência TelE-DdAFelE, editor. Brasília: Ministério da Saúde; 2008. p. 136.

15. Brasil. Política Nacional de Práticas Integrativas e Complementares no SUS PNPIC-SUS. In: Básica SdAàS-DdA, editor. Brasília: Ministério da Saúde; 2006. p. 92.

16. Holtmann G, Adam B, Haag S, Collet W, Grunewald E, Windeck T. Efficacy of artichoke leaf extract in the treatment of patients with functional dyspepsia: a six-week placebo-controlled, double-blind, multicentre trial. Aliment pharmacol ther. 2003;18(11-12):1099-105.

17. Marakis G, Walker AF, Middleton RW, Booth JC, Wright J, Pike DJ. Artichoke leaf extract reduces mild dyspepsia in an open study. Phytomedicine. 2002;9(8):6949.

18. Noldin VF, Cechinel Filho V, Monache FD, Benassi JC, Christmann IL, Pedrosa $\mathrm{RC}$, et al. Composição química e atividades biológicas das folhas de Cynara scolymus L. (alcachofra) cultivada no Brasil. Qim nova. 2003;26(3):331-4.

19. Tsai HH, Lin HW, Simon Pickard A, Tsai HY, Mahady GB. Evaluation of documented drug interactions and contraindications associated with herbs and dietary supplements: a systematic literature review. Int j clin pract. 2012;66(11):1056-78.

20. Amorim MMR, Santos LC. Tratamento da vaginose bacteriana com gel vaginal de Aroeira (Schinus terebinthifolius Raddi): ensaio clínico randomizado. Rev bras ginecol obstet. 2003;25:95-102.

21. Lucena PLH, Ribas-Filho JM, Mazza M, Czeczko NG, Dietz UA, Neto MAC, et al. Avaliação da ação da Aroeira (Schinus terebinthifolius Raddi) na cicatrização de feridas cirúrgicas em bexiga de ratos. Acta cir bras. 2006;21:46-51.

22. Bach DB, Lopes MA. Estudo da viabilidade econômica do cultivo da babosa (aloe vera L.). Ciênc agrotec. 2007;31:1136-44.

23. Oliveira SHS, Soares MJGO, Rocha PS. Uso de cobertura com colágeno e aloe vera no tratamento de ferida isquêmica: estudo de caso. Rev esc enferm usp. 2010;44(2):346-51.

24. Jensen B. Herbal drug interaction chart. Rxfiles; 2003. p. 52-3. 
25. Abebe W. An overview of herbal supplement utilization with particular emphasis on possible interactions with dental drugs and oral manifestations. J dent hyg. 2003;77(1):37-46.

26. Salvi RM, Heuser ED. Interações: medicamentos $x$ fitoterápicos: em busca de uma prescrição racional. Porto Alegre: Edipucrs; 2008. 116 p.

27. Nicoletti MA, Oliveira-Júnior MA, Bertasso CC, Caporossi PY, Tavares APL. Principais interações no uso de medicamentos fitoterápicos. Infarma. 2007;19(1):3240.

28. Reis NT. Nutrição clínica: interações. Rio de Janeiro: Rubio; 2004.

29. Mariot MP, Barbieri RL. O conhecimento popular associado ao uso da espinheira-santa (Maytenus ilicifolia e M. aquifolium). Rev bras biocienc. 2008;5(S1):666-8.

30. Montanari T, Bevilacqua E. Effect of Maytenus ilicifolia Mart. on pregnant mice. Contraception. 2002;65(2):171-5.

31. Rossato AE, Chaves TRC, Rossato AE, Pierini MM, Amaral PA, Santos RR, et al. Fitoterapia racional: aspectos taxonômicos, agroecológicos, etnobotânicos e terapêuticos. Dioesc 2012(1):211.

32. Ferreira $A O$, Brandão M. Guia prático da farmácia magistral. São Paulo: Pharmabooks; 2008.

33. Langmead L, Rampton DS. Review article: herbal treatment in gastrointestinal and liver disease benefits and dangers. Aliment pharmacol ther. 2001;15(9):1239-52.

34. Van Den Bout Van Den Beukel CJ, Koopmans PP, Van Der Ven AJ, De Smet PA, Burger DM. Possible drug-metabolism interactions of medicinal herbs with antiretroviral agents. Drug Metab Rev. 2006;38(3):477-514.

35. Betoni JE, Mantovani RP, Barbosa LN, Di Stasi LC, Fernandes Junior A. Synergism between plant extract and antimicrobial drugs used on Staphylococcus aureus diseases. Mem inst oswaldo cruz. 2006;101(4):387-90.

36. Pilla MAC, Amorozo MCM, Furlan A. Obtenção e uso das plantas medicinais no distrito de Martim Francisco, Município de Mogi-Mirim, SP, Brasil. Acta bot bras. 2006;20(4):789-802.

37. Lima CB, Bellettini NMT, Silva AS, Cheirubim AP, Janani JK, Vieira MAV, et al. Uso de plantas medicinais pela população da zona urbana de Bandeirantes-PR. Rev bras biocienc. 2008;5(S1):600-2. 
38. Akdogan M, Gultekin F, Yontem M. Effect of Mentha piperita (Labiatae) and Mentha spicata (Labiatae) on iron absorption in rats. Toxicol ind health. 2004;20(610):119-22.

39. Almeida FM, Alves MTSSdB, Amaral FMM. Uso de plantas com finalidade medicinal por pessoas vivendo com HIV/ AIDS em terapia antirretroviral. Saude soc. 2012;21:424-34.

40. Nahás EAP, Nahás Neto J, De Luca LA, Traiman P, Pontes A, Dalben I. Efeitos da isoflavona sobre os sintomas climatéricos e o perfil lipídico na mulher em menopausa. Rev bras ginecol obstet. 2003;25(5):337-43.

41. Sousa RL, Filizola RG, Diniz MFFM, Sousa ESS, Moraes JLR. Ensaio clínico placebo-controlado com isoflavonas da soja para sintomas depressivos em mulheres no climatério. Rev bras ginecol obstet. 2006;28:91-100.

42. Marliére LDP, Ribeiro AQ, Brandão MGL, Klein CH, Acurcio FA. Utilização de fitoterápicos por idosos: resultados de um inquérito domiciliar em Belo Horizonte (MG), Brasil. Rev bras farmacogn. 2008;18:754-60.

43. Morris ME, Zhang S. Flavonoid-drug interactions: effects of flavonoids on ABC transporters. Life sci. 2006;78(18):2116-30.

44. Plus M. Psyllium (Plantago ovata, Plantago isphagula) www.nlm.nih.gov: Medline Plus; 2015 [cited 2015]. Available from: https://www.nlm.nih.gov/medlineplus/druginfo/natural/866.html.

45. Fugh-Berman A. Herb-drug interactions. Lancet. 2000;355(9198):134-8.

46. Abebe W. Herbal medication: potential for adverse interactions with analgesic drugs. J clin pharm ther. 2002;27(6):391-401.

47. Vale NB. A farmacobotânica, ainda tem lugar na moderna anestesiologia? Rev bras anestesiol. 2002;52(3):368-80.

48. Nicoletti MA, Carvalho KC, Oliveira Júnior MA, Bertasso CC, Caporossi PY, Tavares APL. Uso popular de medicamentos contendo drogas de origem vegetal e/ou plantas medicinais: principais interações decorrentes. Rev saúde. 2009;4(1):25-39.

49. Akesson C, Lindgren H, Pero RW, Leanderson T, Ivars F. Quinic acid is a biologically active component of the Uncaria tomentosa extract C-Med 100. Int immunopharmacol. 2005;5(1):219-29.

50. Heitzman ME, Neto CC, Winiarz E, Vaisberg AJ, Hammond GB. Ethnobotany, phytochemistry and pharmacology of Uncaria (Rubiaceae). Phytochemistry. 2005;66(1):5-29. 


\section{Revisão}

51. Jurgensen S, Dalbo S, Angers P, Santos AR, Valle RMR. Involvement of 5-HT2 receptors in the antinociceptive effect of Uncaria tomentosa. Pharmacol biochem behav. 2005;81(3):466-77.

52. Kloucek P, Polesny Z, Svobodova B, Vlkova E, Kokoska L. Antibacterial screening of some Peruvian medicinal plants used in Calleria District. J ethnopharmacol. 2005;99(2):309-12.

53. Sheng Y, Akesson C, Holmgren K, Bryngelsson C, Giamapa V, Pero RW. An active ingredient of Cat's Claw water extracts identification and efficacy of quinic acid. J ethnopharmacol. 2005;96(3):577-84.

54. Pilarski R, Zieliński H, Ciesiołka D, Gulewicz K. Antioxidant activity of ethanolic and aqueous extracts of Uncaria tomentosa (Willd.) DC. J ethnopharmacol. $2006 ; 104(1-2): 18-23$.

55. Paiva LCA, Ribeiro RA, Pereira JV, Oliveira NMC. Avaliação clínica e laboratorial do gel da Uncaria tomentosa (Unha de Gato) sobre candidose oral. Rev bras farmacogn. 2009;19:423-8.

56. Ciraulo DA, Shader RI, Greenblatt DJ, Creelman W. Manual de interações medicamentosas em psiquiatria. 3 ed. Porto Alegre: Armed; 2007. 